\title{
Forma tumoral encefálica esquistossomótica: apresentação de um caso tratado cirurgicamente
}

\author{
A tumoral form of encephalic schistosomiasis: report of a case \\ submitted to surgical treatment
}

\author{
Luiz A. Ferreira, Francisco lucio C. Lima, Mario do Rosório 0. \\ dos Anjos e Jackson M. L. Costa
}

\begin{abstract}
Resumo Os relatos da esquistossomose mansônica acometendo o sistema nervoso central (SNC) são pouco frequentes, sendo raramente descrito no encéfalo, onde os ovos do Schistosoma mansoni usualmente provocam reação granulomatosa comportando-se como lesão expansiva. Um paciente do sexo masculino, procedente de São Vicente de Ferrer, Baixada Ocidental Maranhense, área endêmica da doença, 27 anos de idade, apresentou tumor no cerebelo, o qual foi tratado cirurgicamente. O estudo histopatológico da lesão cerebelar, demonstrou extensa reação granulomatosa ao redor de ovos em desintegração de Schistosoma mansoni. Os granulomas exibiam diferentes estágios de evolução, desde necrótico exsudativo até produtivo. Este caso representa o primeiro de neuroesquistossomose relatado no estado do Maranhão. A evolução pósoperatória do paciente caracterizou-se por déficit e incoordenação motora dos membros superior e inferior direito associado a tremores.
\end{abstract}

Palavras-chaves: Forma tumoral. Esquistossomose mansônica. Encéfalo. Estado do Maranhão.

\begin{abstract}
The involvement of the central nervous system in the Schistosomiasis mansoni is uncommon and in the literature exist few reports in relation this ocurrence in the brain, in that the schistosoma eggs, usually promote a granulomatous reaction that remember an expansive tumour. A patient, male sex, from São Vicente de Ferrer (MA), 27 years old, had a tumour in the cerebellum, that had cirurgical therapy. Later histological studies of the cerebellar material of the patient, demonstrated an extensive granulomatous reaction around schistosoma eggs. The granulomatous reaction present different stages of evolution, since degenerative necrosis to organization granulomatous reaction. This is the first report from Maranhão (Brazil). The evolution of the patient was caracterized for incordination of members and trembles.
\end{abstract}

Key-words: Tumoral form. Schistosomiasis mansoni. Cerebellum. Maranhão state.

Núcleo de Patologia e Medicina Social do departamento de Patologia da Faculdade de Medicina da Universidade Federal do Maranhão (UFMA), São Luis, MA ,Brasil.

Endereço para correspondencia: Prof. Luiz A. Ferreira. Dept Patologia/FM/UFMA. Praça Madre Deus 2, 65025-560 São Luis, MA. Fax (098) 222-5135

Recebido para publicação em 26/05/97. 
A esquistossomose, nos seus diversos tipos, é uma parasitose que afeta em torno de 300 milhões de indivíduos em diversos continentes, constituindo-se na segunda maior endemia parasitária do mundo2 1314.

O Schistosoma mansoni é a única espécie existente nas Américas. Inicialmente se fixou nas Antilhas, Guianas, Venezuela e posteriomente, no Brasil. Em nosso país a doença atinge cerca de 10 a 12 milhões de indivíduos, ocorrendo predominantemente em comunidades rurais, com expansão para os centros urbanos, estando intimamente relacionada com precárias condições higiênicas e inadequados recursos sanitários. O Nordeste brasileiro constitui-se na principal área endêmica do país, destacando-se os estados da Bahia, Pernambuco, Minas Gerais, Alagoas e Sergipe como as principais áreas da doença 17.

No Maranhão a esquistossomose mansônica vem sendo observada desde 1920 e constitui relevante problema de saúde pública, apresentando focos em diversas regiões e uma área endêmica, a Baixada Ocidental Maranhense. Esta região caracteriza-se por ter uma fauna e flora constituídas por um misto das regiões Norte e Nordeste, sendo uma das regiões economicamente mais pobres do Maranhão, com a população sobrevivendo as custas dos lagos naturais, da pesca, da caça, lavoura e pecuária de pequeno porte. Dessa forma, o homem da Baixada Maranhense, devido ao seu trabalho, vive em contato permanente com águas contaminadas pelo $S$. mansoni, onde um dos reservatórios é o rato (Holochilus braziliensis nanus Thomas, 1897), constituindo-se a Esquistossomose uma doença ocupacional naquela região1 5 .

Apesar do caráter sistêmico a esquistossomose mansônica no que diz respeito ao comprometimento encefálico é rara, não tendo frequência bem definida, sendo descrito sobretudo na espécie S. japonicum ${ }^{18}$.

O primeiro registro, descrevendo a localização cerebral de ovos de Schistosoma, foi feita por Yamagiwa em 1889. Em breve histórico da neuroesquistossomose, Brito e cols6 atribuem a Müller e Stender em 1930, a primeira descrição de ovos de $S$. mansoni na medula espinhal, num caso de mielite transversa observado em um alemão que havia residido por alguns anos no Brasil, sendo que em nosso país coube a Gama e Sá em 1945 o primeiro relato da retirada de um granuloma esquistossomótico da médula de um paraplégico8.
A literatura é unânime em afirmar que a espécie $S$. japonicum acomete mais freqüentemente o encéfalo, enquanto que o $S$. mansoni e $S$. hematobium prevalecem na medula espinhal2 34.

A deposição dos ovos pode ocorrer em qualquer área do encéfalo ou da médula, levando em geral a formação de granulomas. Contudo, as alterações caracterizam-se desde lesões microscópicas discretas e insuficientes para causar manifestações clínicas até quadros graves de mielite transversa, de tumor medular, quadro de epilepsia, síndromes deficitárias, hipertensão intracraniana e hemorragia cerebral maciça, podendo levar a morte2 19.

O presente trabalho registra o primeiro caso de neuroesquistossomose em paciente proveniente do Estado do Maranhão, sendo de relevante importância a divulgação das manifestações neurológicas e chamar a atenção dos clínicos que trabalham nas áreas endêmicas quanto às manifestações com que se apresenta a doença.

\section{RELATO DO CASO}

P.S.S, 27anos, masculino, branco, solteiro, policial militar, procedente de São Vicente de Ferrer (Baixada Ocidental Maranhense), data da internação 15.06.94. Aproximadamente 4 meses antes apresentou febre acompanhada de cefaléia de leve intensidade, naúseas e vômitos ocasionais, anorexia, emagrecimento, dor epigástrica tipo cólica, assim como episódios diarréicos freqüentes. Internou-se no Hospital Beneficiente da Policia Militar, fazendo tratamento sintomático, sem diagnóstico conclusivo. Permaneceu internado por duas semanas, obtendo relativa melhora dos sintomas e alta hospitalar. Posteriormente, a sintomatologia reapareceu e na mesma ocasião o exame parasitológico de fezes confirmou a presença de ovos de S. mansoni. Fez tratamento orientado pela Fundação Nacional de Saúde (FNS-MA) no próprio município com Oxamaniquine em dose única, ocorrendo remissão da sintomatologia em poucos dias.

Após um mês começou a apresentar cefaléia occipital, inicialmente de leve intensidade, até tornar-se severa, náuseas, vômitos, dificuldade de deambulação e, posteriormente, transtornos visuais. Internou-se em um hospital do interior do Estado por uma semana não havendo melhora, quando então procurou serviço especializado em São Luis (MA). No exame neurológico foi observado síndrome cerebelar à direita, edema de papila bilateral. O exame 
tomográfico do crânio revelou processo tumoral no cerebelo.

Internou-se no Hospital Universitário Presidente Dutra da Universidade Federal do Maranhão (UFMA), para tratamento cirúrgico do referido tumor, com exérese completa do mesmo em 17.06.94. Como antecedentes epidemiológicos o paciente relata banhos e pesca em lagoas de São Vicente de Ferrer (MA), região endêmica de esquistossomose, há cerca de um mês antes do início de sua doença.

Exames laboratoriais e evolução do caso: tomografia axial de crânio (04.06.94) revelou área isodensa captante com halo de edema no hemisfério cerebelar direito com efeito de massa, comprimindo e desviando o IV ventrículo e cisterna quadrigeminal e dilatação moderada dos ventrículos laterais e III ventríulo (Figura 1).

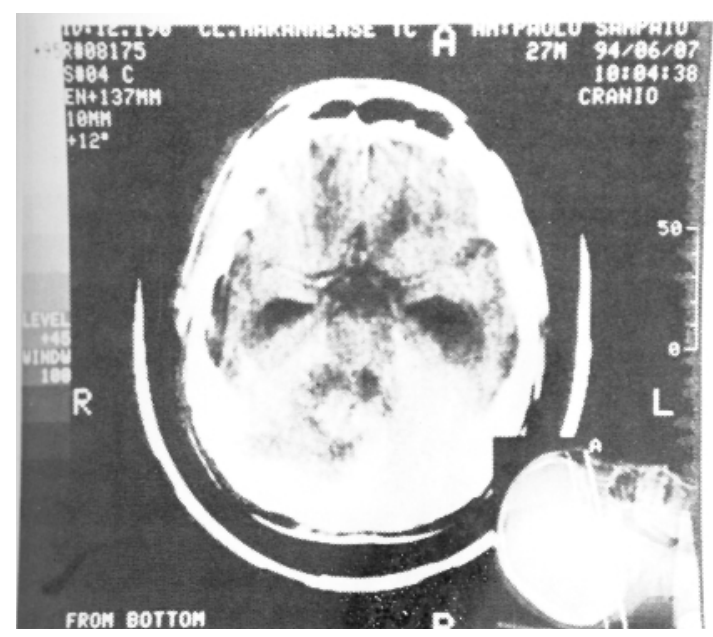

Figura 1 - Tomografia computadorizada de crânio revelando processo tumoral ocupando espaço no hemisfério cerebelar direito.

Biópsia retal (24.08.94): no exame a fresco do fragmento de mucosa retal não se demonstrou ovos de Schistosoma mansoni. Relatório anatomopatológico do material retirado cirurgicamente do cerebelo do paciente: o exame histopatológico revelou secções de fragmentos de tecido nervoso cerebelar exibindo reação inflamatória crônica granulomatosa, com granulomas em diferentes fases de evolução (Figuras 2, 3 e 4). Outras áreas exibiam congestão, focos hemorrágicos e reação glial. Após a cirurgia evoluiu favoravelmente, apresentando

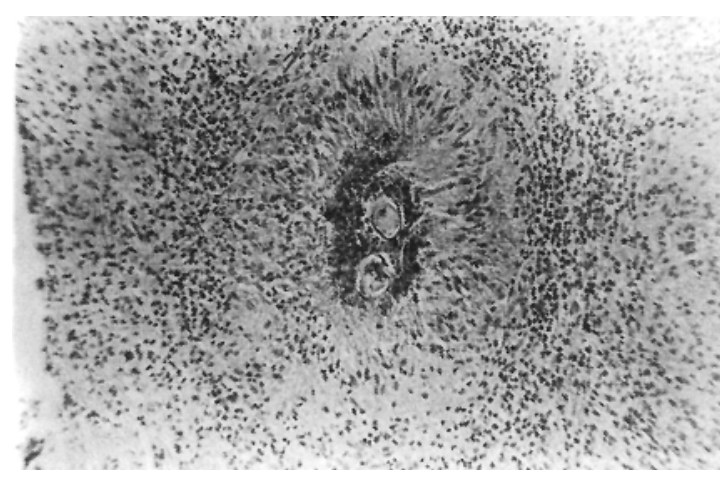

Figura 2 - Granuloma com ovos de S. mansoni envolvidos por necrose, células epitelióides "em paliçada" e, mais externamente, linfócitos, plasmócitos e eosinófilos (granuloma na fase necrótico-exsudativa). ( $H E 40 \times)$.

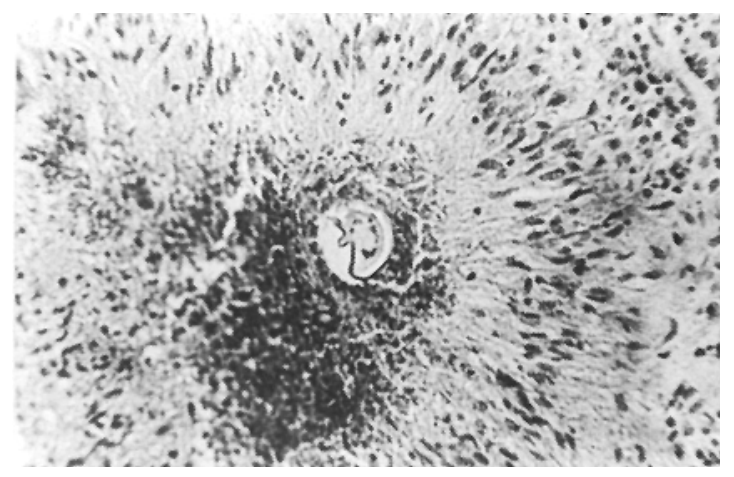

Figura 3 - Granuloma com ovo de S. mansoni envolvido por necrose, células epitelióides "em paliçadas", linfócitos, plasmócitos e eosinófilos (granulomá necrótico-exsudativo), $(40$ x).

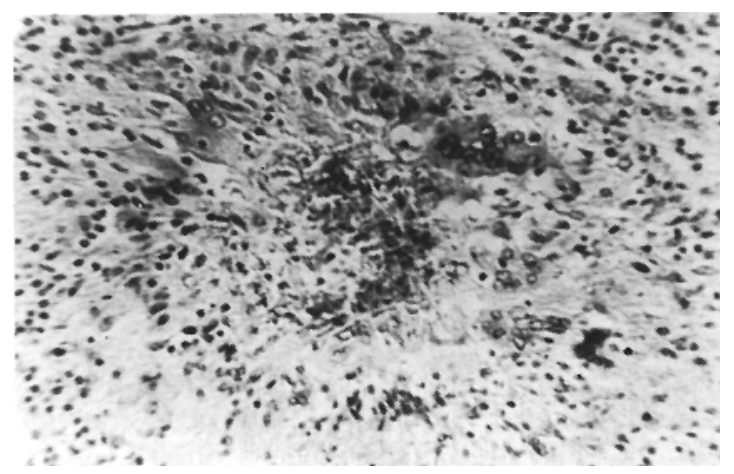

Figura 4 - Granuloma na fase produtiva, constituido por restos de ovos de S. mansoni envolvidos por células epitelióides e gigantes multinucleadas, linfócitos e plasmócitos, (HE 40 X). 
apenas alguns picos febris e episódios de vômitos. Alta hospitalar em 10.07.94, apresentando como sequelas: tremores, déficit e incordenação motora dos membros superior e inferior direitos que persistem até o presente momento.

\section{DISCUSSÃO}

A neuroesquistossomose é considerada rara pela literatura mundial e ainda não tem sua frequência totalmente estabelecida, principalmente pela falta de comprovação histológica em grande número de casos publicados. Contudo existe extensa literatura a respeito do assunto, desde o primeiro caso histologicamente comprovado há mais de um século6 79.

Em 1963, Marcial-Rojas e cols12, após revisar a literatura mundial, encontraram 97 casos de envolvimento de SNC por Schistosoma, sendo 26 por S. mansoni, dos quais 17 envolvendo a médula espinhal e 9 o cérebro. Nesse mesmo estudo os autores demonstraram a correlação entre o local de acometimento do sistema nervoso com a espécie parasitária. $O S$. japonicum é responsável pela maioria dos casos relatados e prevalece no encéfalo, enquanto $S$. mansoni e S. haematobium prevalecem na médula. Acredita-se que a predominância do $S$. japonicum nas lesões neurológicas, deve-se ao fato de que a fêmea desta espécie libera um número maior de ovos que as outras espécies.

Queirós17 em 1974 ao realizar necrópsias em 10 pacientes esquistossomóticos encontrou ovos do parasita no SNC de todos eles, contudo apenas um paciente apresentava sintomatologia neurológica. Pitella e Lana-Peixoto15 16 em 1981, demonstraram que $26 \%$ de 46 pacientes com esquistossomose hepatoesplênica apresentavam extensa deposição de ovos no cerebro, somente um com sintomas neurológicos. Estes estudos de necrópsia mostraram que o envolvimento do SNC na esquistossomose mansônica parece ser mais frequente do que se acredita, mesmo que apenas uma minoria de casos apresente sintomatologia neurológica.

Acredita-se que o Schistosoma mansoni alcance o SNC através de vários mecanismos. $\mathrm{Na}$ primeira, os ovos são conduzidos através dos plexos venosos de Batson, onde existem anastomoses desprovidas de válvulas entre as veias pélvicas e os plexos perimedulares, podendo então alcançar a médula e o cérebro. Isto pode ser facilitado pelo aumento da pressão intra-abdominal. A grande incidência de mielopatia acometendo a região lombossacra seria explicada por via de chegada. Ou então, os vermes adultos migrariam de maneira anômala, depositando grande quantidade de ovos no interior dos vasos do sistema nervoso. Esta hipótese é favorecida pelo encontro de ovos enfileirados e vermes adultos no tecido nervoso e no interior dos vasos perimedulares. A vigência de hipertensão pulmonar e comunicação cardíaca direita-esquerda também pode ser causa de embolização de ovos do Schistosoma até o SNC10 1120.

Os ovos podem acumular-se em determinadas áreas do SNC e produzir uma reação ao seu redor suficiente para formar numerosos granulomas que agirão como lesão expansiva, como foi observado em nosso caso.

Ao que tudo indica, na neuroesquistossomose como nas demais formas da parasitose participam os dois mecanismos imunitários, o celular e o humoral. A formação de granulomas é determinada por reação celular do tipo hipersensibilidade tardia. As lesões produzidas pelo parasita dependem do grau de infestação e da resposta imunológica, geral e local do hospedeiro. Isso sugere que as pessoas parasitadas não residentes em áreas endêmicas estão mais propensas a desenvolver formas agudas mais graves. Em relação ao caso descrito, embora o paciente procedesse de área endêmica, a ocorrência de sintomas bastante sugestivos de esquistossomose aguda, leva a crer contato recente do mesmo com o $S$. mansoni8 1219.

As manifestações neurológicas usualmente apresentam-se na ausência de qualquer outra manifestação da doença e, em poucos casos, são precedidas de manifestações agudas da esquistossomose. O diagnóstico de neuroesquistosomose deve ser suspeitado em pacientes que possam ter tido contato com o parasita em área endêmica e que apresentam manifestação neurológica encefálica, medular ou meníngea, onde não se encontrou outra causa. A tomografia computadorizada do crânio pode ser valiosa ao localizar lesões expansivas que podem corresponder a granulomas. $O$ exame histopatológico do material ressecado cirurgicamente, mostrando o granuloma circundando o ovo de Schistosoma é a única prova de certeza como observado em nosso caso17 20. 
No que diz respeito à terapêutica da neuroesquistossomose os autores usam de um modo geral corticosteróides, sobretudo dexametasona e predinisona, associados com os esquistossomicidas oxamaniquine ou praziquentel. O tratamento cirúrgico, seguido de tratamento clínico antiesquistossomótico, tem sido feito no caso de granulomas cerebrais ou cerebelares, consistindo na excisão dos mesmos. No presente caso, o tratamento foi cirúrgico, conduta considerada correta, mesmo sem que houvesse a suspeita inicial de neuroesquistossomose, levando a possibilidade de tratamento clínico imediato.

\section{REFERÊNCIAS BIBLIOGRÁFICAS}

1. Alvim AC. A esquistossomose no Maranhão. Hiléia Médica 2:151-157, 1980.

2. Andrade AN. Neuroesquistossomose. Arquivo de Neuro-Psiquiatria 44:275 -279, 1986.

3. Bambirra EA, Andrade JS, Cesarini I, Rodrigues PA, Drumond CASA. The tumoral form of schistosomiasis: report of a case with cerebellar involvement. American Journal of Tropical Medicine and Hygiene 33:76-79, 1984.

4. Bléry M, Chagnon S, Sagui M. Bilharziose cérébrale. Aspect tomodensitométrique. A propos d'une observation. Journal du Radiologie 66:141-142, 1985.

5. Borgeaud TV, Lemos Neto RC, Peter F, Bastos OC. Constatações sobre a importância dos roedores silvestres (Holochilus brasiliensis nanus, Thomas, 1897) a epidemiologia da esquistossomose mansônica própria da pré-amazonia, MaranhãoBrasil. Cadernos de Pesquisa da Universidade Federal do Maranhão 2:86-99, 1986.

6. Brito JCF, Silva JAG, Silva EB, Viana NO Neuroesquistossomose Medular. Arquivo de Neuropsiquiatria 50:207-211,1992.

7. Costa RO, Gameleira FT, Tenório RB, Brás LH, Costa VB, Pinto Junior JM. Neuroesquistossomose em Alagoas. Revista Brasileira de Neurologia 28:79-84, 1992.

8. Gama C, Sá JM. Esquistossomose medular. Granulomas produzidos por ovos de Shistossoma mansoni comprimindo a médula, epicone, cone e cauda equina. Arquivos de Neuro-Psiquiatria 3, 334.

9. Gjerde JO, Mörk S, Larsen JL, Huldt G, Skeidsvoll H, Aarli JA. Cerebral schistosomiasis presenting as a brain tumour. European Neurology. 23:229-236, 1984.
10. Goasguen J, Antoine HM, Saliou P, Herbellau T, Putz DM, Jallon PM, Bacquet C. Bilharziose Cérébrale a Schistosoma mansoni. Revue Neurologique 140:293295, 1984.

11. Livramento JA, Machado LR, Silva LC, Spina-França A. Síndrome do líquido cefalorraqueano na neuroesquistossomose. Arquivos de NeuroPsiquiatria 43:372-377, 1985.

12. Livramento JA, Machado LR, Spina-França A. Sinalização do líquido cefalorraqueano em doenças inflamatórias crônicas do sistema nervoso central. Arquivos de Neuro-Psiquiatria. 44: 351-358, 1986.

13. Marcial-Rojas RA, Fiol RE. Neurological complications of schistosomiasis. Review of the literature and report of two cases of transverse myelitis due to $S$. mansoni. Annals of Internal Medicine 59:215-230, 1963.

14. Ministério da Saúde. Fundação Nacional de Saúde. Coordenação Regional do Maranhão. Programa de controle da esquistossomose 1993.

15. Organização Mundial de Saúde. Control de la esquistosomiasis: Série informes técnicos. Ginebra. 728:33-37, 1985

16. Pittela JEH. Vascular changes in cerebral schistosomiasis mansoni: a histopatological study of fifteen cases. The American Journal of Tropical Medicine and Hygiene 34:898-902, 1985.

17. Pittela JEH. The relation between involvement of the central nervous system in schistosomiasis mansoni and the clinical forms of the parasitosis. A Review . Journal of Tropical Medicine and Hygiene 94:15-21, 1991.

18. Queiros AC. O envolvimento do sistema nervoso central na esquistossomose mansonica. Revista de Patologia Tropical 3:255-261, 1974.

19. Sabatier P, Saliou P, Rougier Y, Schill H, Goasguen J, Antoine HM. Pseudo-Tumeur cérébrale, manifestation isoleé D'une Bilharziose a Schistosoma mansoni chez un Européen. Bulletin Societe Pathologie Exotique 76:509-513, 1983.

20. Scringeour E M, Gajdusek CD. Involvement of the central nervous system in Schistosoma mansoni and S. haematobium infection. Brain 108:10231038,1985 .

21. Sztajnberg MC, Fernandes RCL. Mielite esquistossomótica. Relato de um caso tratado com praziquantel . Revista Brasileira de Neurologia 21:6166,1985 